\title{
Graph-TSP from Steiner Cycles
}

\author{
Satoru Iwata ${ }^{1}$, Alantha Newman ${ }^{2}$, and R. Ravi ${ }^{3}$ \\ 1 Department of Mathematical Informatics, University of Tokyo, Tokyo 113-8656, \\ Japan. Email: iwata@mist.i.u-tokyo.ac.jp \\ 2 CNRS-Université Grenoble Alpes and G-SCOP, F-38000 Grenoble, France. Email: \\ alantha.newman@grenoble-inp.fr \\ 3 Tepper School of Business, Carnegie Mellon University, USA. Supported in part by \\ NSF grants CCF1143998 and CCF1218382. Email: ravi@cmu.edu
}

\begin{abstract}
We present an approach for the traveling salesman problem with graph metric based on Steiner cycles. A Steiner cycle is a cycle that is required to contain some specified subset of vertices. For a graph $G$, if we can find a spanning tree $T$ and a simple cycle that contains the vertices with odd-degree in $T$, then we show how to combine the classic "double spanning tree" algorithm with Christofides' algorithm to obtain a TSP tour of length at most $\frac{4 n}{3}$. We use this approach to show that a graph containing a Hamiltonian path has a TSP tour of length at most $4 n / 3$.

Since a Hamiltonian path is a spanning tree with two leaves, this motivates the question of whether or not a graph containing a spanning tree with few leaves has a short TSP tour. The recent techniques of Mömke and Svensson imply that a graph containing a depth-first-search tree with $k$ leaves has a TSP tour of length $4 n / 3+O(k)$. Using our approach, we can show that a $2(k-1)$-vertex connected graph that contains a spanning tree with at most $k$ leaves has a TSP tour of length $4 n / 3$. We also explore other conditions under which our approach results in a short tour.
\end{abstract}

\section{Introduction}

We consider the well studied Traveling Salesman problem with graph metric, also known as graph-TSP. Throughout this paper, the input graph $G=(V, E)$ is assumed to be an undirected, unweighted, 2-(vertex) connected graph, and all edge lengths in the complete graph can be obtained via the shortest path metric on the given graph. Our goal is to find a tour of minimium length that visits each vertex at least once. In this paper, we focus on a connection between graph-TSP and that of finding Steiner cycles.

\subsection{Background}

Graph-TSP has received much attention recently. Oveis Gharan, Saberi and Singh were the first to improve on the approximation ratio of $3 / 2$ by an infinitesmial, but constant, factor [12]. This was quickly followed by the breakthrough work of Mömke and Svensson, who introduced a new approach leading 


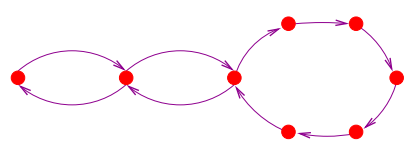

Fig. 1. In this (non-simple) cycle $C$, the number of unique vertices $|C|=8$, but the length of the cycle $\ell(C)=10$.

to a substantial improvement in the approximation ratio [19]. Subsequently, Mucha gave a refined analysis of their approach, proving an approximation ratio of 13/9 for graph-TSP [20]. More recently, Sebő and Vygen presented an approximation algorithm with ratio $7 / 5$ for the problem [23].

It is widely believed that an approximation ratio of at most $4 / 3$ should be efficiently computable. The approach of Mömke and Svensson is based on setting up a circulation network and showing that a low-cost circulation leads to a low cost TSP tour. They obtained a 4/3-approximation for subcubic graphs, but high-degree graphs appear to be more challenging for their framework. Vishnoi recently gave a randomized algorithm that finds a TSP tour very close to $n$ with high probability for a $k$-regular graph when $k$ is sufficiently large [26]. Our goal is to consider other techniques that are applicable for graphs that are not low-degree or regular.

\section{Steiner Cycles}

The Steiner cycle problem has been previously, but not extensively, studied under varying definitions $[8,13,25]$. For our purposes, a Steiner cycle is defined to be a simple cycle that contains a specified subset $S \subseteq V$ of vertices. It may also contain any subset of vertices from the set $V \backslash S$. We use the following definition:

Definition 1. Given a graph $G=(V, E)$ and a subset of vertices, $S \subseteq V$, a Steiner cycle, $C \subset E$, is a simple cycle whose vertices contains the set $S$.

It is important to observe that in our definition of a Steiner cycle, there are no repeated vertices, since a Steiner cycle is a simple cycle. We define an approximate Steiner cycle as one in which we are allowed to repeat vertices. For a cycle $C$, we will use $|C|$ to denote the number of unique vertices it contains. We define the cycle length $\ell(C)$, to be total length of a traversal of the cycle. If $C$ is a simple cycle, then $|C|=\ell(C)$. For example, in Figure 1, the non-simple cycle has eight unique vertices and has length ten. Our definition of cycle length is the same as the standard definition for the length of a TSP tour in the graph metric. Now we can define an approximate Steiner cycle.

Definition 2. Given a graph $G=(V, E)$ and a subset of vertices, $S \subseteq V$, an approximate Steiner cycle, $C \subset E$, with relative length $\beta \geq 1$ is a cycle whose vertices contains the set $S$ and for which $\ell(C) /|C| \leq \beta$. 
In an approximate Steiner cycle, since we are allowed to repeatedly visit vertices as we traverse the cycle, it may be the case that the number of unique vertices will be smaller than the length, $|C|<\ell(C)$. Throughout this paper, whenever we refer simply to a "cycle", we mean a simple cycle.

Other natural definitions of the Steiner cycle problem are concerned with such aspects as minimizing the number of non-required (Steiner) vertices in the cycle. In our definition of the approximate Steiner cycle problem, the only objective that we wish to minimize is the ratio of the length of a cycle, $\ell(C)$, to the number of unique vertices, $|C|$, it contains. Thus, the measure of an optimal solution is independent of the size of the set of required vertices. The work that appears to be most related to the Steiner cycle problem as we have defined it concerns the concept of cyclability: A set of vertices $X \subseteq V$ is called cyclable if it is contained in some cycle. The quantity $\operatorname{cyc}(G)$ is the maximum number such that all subsets containing at most $\operatorname{cyc}(G)$ vertices are cyclable. Note that $\operatorname{cyc}(G)=n$ if and only $G$ is Hamiltonian. It seems that most of the work on cyclability has been done with the intention of eventually using it to prove that certain graphs are Hamiltonian or because it can be viewed as a relaxation of Hamiltonicity. An interesting list of theorems on cyclability can be found in [21]. Here, we explore cyclability as a tool to obtain approximate TSP tours.

\subsection{Our Approach}

Graph-TSP can clearly be cast as a special case of the Steiner cycle problem in which all of the vertices in $V$ are required to belong to the Steiner cycle. In this paper, we show that even if the required set of vertices is possibly much smaller than the entire vertex set $V$, an (approximation) algorithm for the Steiner cycle problem can still be used to approximate graph-TSP.

Suppose we can find a spanning tree $T$ for the graph $G$ and a simple cycle $C_{T}$ that contains all of the vertices that have an odd-degree in the tree $T$. When $\left|C_{T}\right|$ is large, we show that we can use the folklore "double spanning tree" algorithm to find a short tour. When $\left|C_{T}\right|$ is small, then there is a small matching on the odddegree vertices in $T$ and we can therefore show that Christofides algorithm [5] yields a short tour. Thus, our algorithm, described in Section 3, can be viewed as a combination of these two standard algorithms for graph-TSP.

We are not aware of any previous work studying how to combine these two classic algorithms for graph-TSP. However, a similar algorithm that combines these two algorithms was given by Guttman-Beck, Hassin, Khuller and Raghavachari for the $s, t$-path TSP [15]. In their algorithm, they first find an MST for the input graph. If the path from $s$ to $t$ in this MST is long, they double edges in the MST that do not belong to this path. If the path from $s$ to $t$ is short, they modify the input graph by adding an edge from $s$ to $t$ with length equal to the shortest $s, t$-path in $G$ and run Christofides on this modified graph as in the algorithm by Hoogeveen [17]. Taking the better of these two algorithms results in a 5/3-approximation for the $s, t$-path problem, which does not improve on the worst-case approximation ratio of Hoogeveen's algorithm. Nevertheless, this approach was used to design algorithms for special variants of 

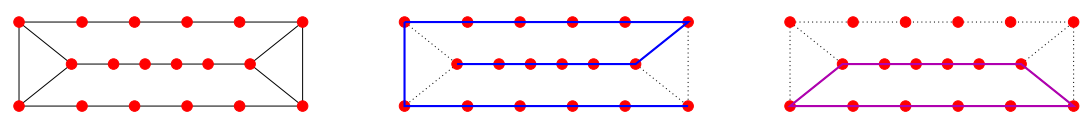

Fig. 2. A graph $G$ with a spanning tree $T$ (second figure, blue edges) and a simple cycle $C_{T}$ (third figure, purple edges) containing all of the odd-degree nodes of $T$.

the path TSP problem [15], and the ideas were also eventually used to obtain improved approximation guarantees for the $s, t$-path TSP itself [22]. In our algorithm, rather than basing the subcases on the path length from $s$ to $t$ in an MST, we are basing the two subcases on the length of a cycle containing the nodes with odd degree in a particular MST.

\subsection{Overview of our Results}

In Section 3, we give a complete description of our algorithm. In Section 4, we use this algorithm to show that if the input graph contains a Hamiltonian path, then it has a TSP tour of length at most $4 n / 3$. Moreover, if we are given the Hamiltonian path, then we can efficiently find such a tour. This theorem was first proved by Gupta using a different approach [14].

One can view a Hamiltonian path as a spanning tree with two leaves. A natural question is how well we can approximate a TSP tour in a graph that contains a spanning tree with few leaves. In Section 5, we show how our approach can be used to address this question in some special cases. In Section 6 , we discuss how approximate Steiner cycles can also be used to obtain an approximation guarantee for graph-TSP. Finally, in Section 7, we consider some examples.

\section{$3 \quad$ TSP Tours from Steiner Cycles}

Given an undirected, unweighted graph, $G=(V, E)$, with graph metric, our goal is to find a TSP tour of minimum length. A TSP tour must visit each vertex at least once. As stated previously in the introduction, we assume that $G$ is a 2-connected graph and we define $n=|V|$.

Let $T$ be a spanning tree of $G$ and let $S_{T} \subset V$ be the vertices that have odd degree in $T$. Suppose there is a simple cycle $C_{T}$ that contains all the vertices in $S_{T}$. Note that the simple cycle $C_{T}$ can be of arbitrary length, i.e. can contain arbitrarily many vertices in $V \backslash S_{T}$.

Theorem 1. For a given graph $G$, suppose we have a minimum spanning tree $T$ and a simple cycle $C_{T}$ that contains all vertices with odd degree in $T$. Then we can construct a TSP tour of $G$ with length at most $4 n / 3$.

Proof: Consider the following cases. Recall that $\left|C_{T}\right|$ denotes the number of unique vertices contained in the cycle $C_{T}$. Since $C_{T}$ is a simple cycle, $\left|C_{T}\right|$ also denotes its length. 
(i) $\left|C_{T}\right|>2 n / 3$. In this case, we can contract the cycle $C_{T}$ to a single vertex. The resulting graph has at most most $n / 3$ vertices. We can then find a minimum spanning tree on this graph and double each edge. When we uncontract the vertex corresponding to the cycle $C_{T}$, we obtain an Eulerian tour whose total length is at most $4 n / 3$.

(ii) $\left|C_{T}\right| \leq 2 n / 3$. In this case, since all of the vertices of $S_{T}$ are contained in $C_{T}$, there is a matching of the vertices in $S_{T}$ with length at most $n / 3$. Using this matching plus $T$, we obtain an Eulerian tour of $G$ of length at most $4 n / 3$.

We can therefore see that if $G$ has a tree $T$ and a simple cycle $C_{T}$ that contains all of the vertices with odd degree in $T$, then $G$ has a TSP tour of length at most $4 n / 3$. We now show how to apply this theorem to some special classes of graphs.

\section{Graphs Containing a Hamiltonian Path}

Recall that a Hamiltonian path in $G$ is a path that visits each vertex in $V$ exactly once. Note that the first and last vertices on the path might not be adjacent vertices in $G$. More generally, $G$ might not be Hamiltonian. In this section, we show that for an unweighted graph $G=(V, E)$ with graph metric, if $G$ contains a Hamiltonian path, then $G$ has a TSP tour of length at most $4 n / 3$.

Theorem 2. Suppose $G$ contains a Hamiltonian path. Then $G$ has a TSP tour of length at most $4 n / 3$.

Proof: Suppose that the first and last vertices of the Hamiltonian path are adjacent in the graph. Then $G$ is Hamiltonian and, moreover, given the Hamiltonian path, we can find this tour.

If the first and last vertices of the Hamiltonian path are not adjacent in $G$, then since $G$ is 2-vertex connected, we can use Menger's theorem [18, 10], which states that there are two vertex disjoint paths between any two non-adjacent vertices in a 2-vertex connected graph. Thus, we have a simple cycle including the odd-degree nodes on the tree (the first and last nodes in the Hamiltonian path) and the proof of the theorem follows directly from applying Theorem 1.

Since there are constructive proofs of Menger's Theorem, Theorem 2 results in an efficient algorithm, assuming the Hamiltonian path is given.

\section{Graphs Containing a Spanning Tree with $k$ Leaves}

A Hamiltonian path can be viewed as a spanning tree with two leaves. A natural extension is to ask what happens when a graph does not contain a Hamiltonian path but rather a spanning tree with few leaves. Does it still have a short TSP tour? Suppose $G$ has a spanning tree with $k$ leaves. If $G$ is well-connected, we can use a well-known theorem of Dirac to obtain an upper bound on the length of a TSP tour of $G$. 
Theorem 3. Suppose $G$ is 2(k-1)-connected and contains a spanning tree with $k$ leaves. Then $G$ has a TSP tour of length at most $4 n / 3$.

Proof: A spanning tree with $k$ leaves contains at most $2(k-1)$ vertices with odd degree. A theorem of Dirac states that if a graph is $c$-vertex connected, then any subset $X \subseteq V$ of vertices with $|X| \leq c$ is contained in some simple cycle $[9, ?]$. Thus, if $c=2(k-1)$, then $G$ is $c$-connected by the assumption of the theorem. Moreover, $G$ has at most $c$ odd-degree vertices if it has $k$ leaves. We can therefore let $X$ be the set of odd-degree vertices and the theorem follows directly from applying Theorem 1 .

Finding a simple cycle containing $c$ vertices in a $c$-connected graph can be done efficiently (see Chapter 9 in [3]). Thus, Theorem 3 results in an efficient algorithm assuming the spanning with $k$ leaves is given.

More generally, Steiner cycles have been studied by the Graph Theory community and if a set of vertices $X \subseteq V$ is contained in a cycle, then the set $X$ is called cyclable. This terminology is attributed to Chvatal [6]. Moreover, cyclability of a graph $G$, i.e. $\operatorname{cyc}(G)$, is the maximum number such that every subset of at most $\operatorname{cyc}(G)$ vertices is cyclable. If a graph $G$ has a cyclable number $c=\operatorname{cyc}(G)$ and it also contains a spanning tree with at most $c / 2+1$ leaves, then this spanning tree contains at most $c$ odd-degree vertices. Thus, it will contain a TSP tour of length $4 n / 3$ via Theorem 1 . Considerable effort has been invested in computing the cyclablity of certain graph classes. For example, we cite the following two theorems:

Theorem 4. [16] For every 3-connected cubic graph $G$, cyc $(G) \geq 9$. This bound is sharp (the Petersen graph).

Theorem 5. [2] For every 3-connected cubic planar graph $G, \operatorname{cyc}(G) \geq 23$. This bound is sharp.

Theorem 4 implies that if a 3-connected, cubic graph $G$ contains a spanning tree with at most five leaves, then $G$ has a TSP tour of length at most $4 n / 3$. Theorem 5 shows that if a 3 -connected, planar, cubic graph $G$ contains a spanning tree with at most 12 leaves, then $G$ has a TSP tour of length at most $4 n / 3$. We remark that showing that a 3 -connected cubic graph has a spanning trees with at most five leaves as a means to bounding the length of a TSP tour would only be an alternative approach, as it is already known that a cubic graph has a TSP tour of length at most $4 n / 3[1,14,4,19]$.

A well-known theorem of Dirac states that every graph with minimum degree at least $n / 2$ is Hamiltonian. A analogous theorem can be shown for cyclability. Let $X \subseteq V$ be a subset of vertices and define $\sigma_{2}(X):=\min \left\{\sum_{y \in Y} d(y): Y \subseteq\right.$ $X,|Y|=2, Y$ is an independent set $\}$. In other words, if we choose each pair of non-adjacent vertices in $X$ and add up their degrees, $\sigma_{2}(X)$ is the minimum of this quantity. This is used in the following theorem due to Shi:

Theorem 6. [24] Let $G=(V, E)$ be a 2-connected graph and $X \subset V$. If $\sigma_{2}(X) \geq n$, then $X$ is cyclable in $G$. 
If we find a spanning tree $T$ such that all non-adjacent pairs of vertices with odd-degree in $T$ have total degree at least $n$ (in $G$ ), then $G$ has a TSP tour of length at most $4 n / 3$. The vertices that have an even degree in the tree are allowed to have low degree in $G$. Another nice theorem on cyclability is due to Fournier:

Theorem 7. [11] Let $G$ be a 2-connected graph and $X \subseteq V$. If $\alpha(X) \leq \kappa(G)$, then $X$ is cyclable in $G$.

Here, $\alpha(X)$ means the largest independent set in $X$, and $\kappa(G)$ is the connectivity of $G$. It is known that if $\alpha(G) \leq \kappa(G)$, then $G$ is Hamiltonian [7]. Theorem 7 implies that if the set of odd-degree vertices in a spanning tree has a maximum independent set that is smaller than the connectivity of $G$, then $G$ has a TSP tour of length at most $4 n / 3$.

In relation to Theorem 3, it is reasonable to ask if, for sufficiently large $k$, a $2(k-1)$-connected graph has a spanning tree with $k$ leaves. This is not the case as demonstrated by the following example. Consider the complete bipartite graph $G=K_{c, n}$ where $n>>c$. Then $G$ is $c$-connected, but the minimum length TSP tour is roughly $2 n$. So $G$ cannot contain a spanning tree with at most $c / 2+1$ leaves.

\subsection{Graphs Containing a $k$-Leaf DFS Spanning Tree}

If $G$ has a depth-first-search (DFS) spanning tree with $k$ leaves, then we note that the techniques of Mömke and Svensson [19] can be used to obtain a TSP tour of length at most $4 n / 3+2 k / 3$. Specifically, in this case, it is not difficult to see that there is a circulation (as defined by Mömke and Svensson) of cost at most $k$. This implies that one can also use the techniques from Mömke and Svensson to prove Theorem 2. We emphasize that a DFS spanning tree must be used to directly apply the techniques of Mömke and Svensson. In comparison, in Theorem 3, we can use any spanning tree with $k$ leaves. The proof of Lemma 1 is straightforward, but we include it for the sake of completeness.

Lemma 1. If $G$ has a DFS spanning tree with at most $k$ leaves, then it has a circulation, as defined by Mömke and Svensson [19], of cost at most $k$.

Proof: We will demonstrate a 2-connected subgraph of $G$ such that the cost of a circulation on this subgraph is at most $k$.

Consider a path from the root of the DFS tree to a leaf. Let us call this path $p_{1}$. Suppose that the vertices on $p_{1}$ are labeled sequentially from the root to the leaf in increasing order, $1,2, \ldots \ell\left(p_{1}\right)$, where $\ell\left(p_{1}\right)$ denotes the number of vertices in the path $p_{1}$. We find a back-edge from the leaf or the vertex labeled $\ell\left(p_{1}\right)$ to a vertex with the smallest label. Suppose that this edge goes from $\ell\left(p_{1}\right)$ to $h$. Then at the next step, we find the back-edge $(i, j)$ where $\ell\left(p_{1}\right)>i>h$ and $j<i$ and $j$ is as small as possible. Since $G$ is 2-connected, we will always be able to find such an edge. Otherwise $G$ would contain a cut vertex, which would contradict the 2-connectivity of $G$. 
Now consider a path on the DFS tree from some vertex on $p_{1}$ to another leaf. Call the path from the root to this leaf $p_{2}$. Perform the same procedure as above: starting at the leaf, find some back-edges, so that the resulting subgraph containing paths $p_{1}$ and $p_{2}$ and these back-edges is 2-connected. At some point, we will add a back edge that intersects with the path $p_{1}$. If this is a branching node, i.e. the last node that belongs to both $p_{1}$ and $p_{2}$, we will add one more back edge so that the resulting subgraph is 2-connected.

Note that each vertex in $p_{2}$ that is below this branching node, i.e. has a higher label, has only one back-dge coming into it. The only vertices that may have more than one back-edge coming into them are the branch node and another node with a lower label. However, since in Lemma 4.1 of [19], each subtree of a branch node is accounted separately in the circulation network, if the branch node now has, say, two back-edges, it also has two subtrees, so its contribution to the circulation is still zero. A node above the branch node with $B$ back-edges coming into it will contribute at most $B-1$ to the cost of the circulation.

As we add each root-leaf path in the DFS tree, and we add the new path and a set of back-edges to make the subgraph 2-connected, we will add at most one back-edge to a vertex that already has incoming back-edges. Thus, the circulation is upper bounded by $k$ if the DFS tree has $k$ leaves.

Theorem 8. If $G$ has a DFS spanning tree with at most $k$ leaves, then it has a TSP tour of at most $4 n / 3+2 k / 3$.

Proof: This follows from Lemma 1 and Lemma 4.1 of Mömke and Svensson [19].

\section{Cycle Length and Approximation Ratio Tradeoff}

We have shown that a simple cycle that contains the odd-degree nodes in some spanning tree yields a TSP tour of length at most $4 n / 3$. Suppose we can only obtain an approximate Steiner cycle. Then what is the guarantee on the length of the TSP tour? We now show that we can obtain the following tradeoff. For a cycle $C$ in $G$ that is not necessarily simple, recall that $|C|$ is the number of unique vertices in the cycle $C$ and $\ell(C)$ denotes its length.

Theorem 9. Given $G$, a minimum spanning tree $T$ and an approximate Steiner cycle $C_{T}$ that contains all the odd-degree vertices in $T$ such that $\ell\left(C_{T}\right) \leq(1+$ $\gamma)\left|C_{T}\right|$, we can construct a TSP tour of $G$ of length at most $\frac{4 n}{3-\gamma}$.

Proof: We consider two cases based on the number of unique vertices in the cycle $C_{T}$ :

(i) $\left|C_{T}\right|>\frac{2 n}{3-\gamma}$. Then we contract the cycle $C_{T}$ to a single vertex, find a minimum spanning tree on the resulting graph and double each edge in this spanning tree. Since the length of cycle $\ell\left(C_{T}\right) \leq(1+\gamma)\left|C_{T}\right|$, the total length 
of the resulting Eulerian tour is at most:

$$
\begin{aligned}
\ell(T S P) & \leq(1+\gamma)\left|C_{T}\right|+2\left(n-\left|C_{T}\right|\right) \\
& =2 n+(1+\gamma-2)\left|C_{T}\right| \\
& =2 n-(1-\gamma)\left|C_{T}\right| \\
& <2 n-\frac{2 n}{(3-\gamma)}(1-\gamma) \\
& =\frac{4 n}{3-\gamma} .
\end{aligned}
$$

(ii) $\left|C_{T}\right| \leq \frac{2 n}{3-\gamma}$. In this case, we find a matching of the odd-degree vertices in $T$ with length at most $(1+\gamma)\left|C_{T}\right| / 2$. The total length of the resulting Eulerian tour $S$ is at most:

$$
\begin{aligned}
\ell(T S P) & \leq n+(1+\gamma) \frac{\left|C_{T}\right|}{2} \\
& \leq n+\frac{(1+\gamma)}{2} \frac{2 n}{(3-\gamma)} \\
& =\frac{4 n}{3-\gamma} .
\end{aligned}
$$

\subsection{Approximation Guarantees from LP Bounds}

In general, it could be the case that there does not exist a spanning tree whose odd-degree vertices can be contained in a simple cycle. An example of such a graph can be found in Figure 3. However, suppose we can compute, via an LP relaxation or some other means, a lower bound on the length of a TSP tour, e.g. $O P T \geq(1+\alpha) n$ for $0 \leq \alpha \leq 1$. Then the following Corollary of Theorem 9 states a sufficient condition for a $\frac{4}{3}$-approximation to the optimal TSP tour.

Corollary 1. If an optimal tour is lowerbounded by $O P T \geq(1+\alpha) n$ and $G$ contains a spanning tree $T$ and a cycle $C_{T}$ containing the odd-degree nodes of $T$ such that $\ell\left(C_{T}\right) \leq(1+4 \alpha)\left|C_{T}\right| /(1+\alpha)$, then $G$ has a TSP tour of length at most $\frac{4}{3} \cdot O P T$.

Note that Theorem 9 says that if we can find a tree $T$ and a cycle $C_{T}$ such that $\ell\left(C_{T}\right) /\left|C_{T}\right|<4 / 3$, then we can find a TSP tour less than $3 n / 2$. To find a tour shorter than $7 n / 5$ (which is currently the best known bound when the solution to the standard LP relaxation equals $n$ [23]), we require that $\ell\left(C_{T}\right) /\left|C_{T}\right|<8 / 7$.

\section{Discussion}

We have reduced the problem of finding a short TSP tour to the problem of finding an (approximate) Steiner cycle where the required vertices are the odddegree nodes in some spanning tree, and we have flexibility as to whether or not we include the non-required vertices in the cycle. But is this problem any easier 


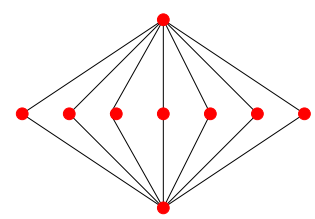

Fig. 3. Any spanning tree of this graph has too many leaves to be spanned by a simple cycle. However, note that the solution to the Held-Karp LP relaxation will be $|E|=2 n$ for this graph, certifying that the lower bound is much greater than $4 n / 3$ in this case.

than graph-TSP itself? For example, in Figure 4, we give an example of a graph and a spanning tree such that the odd-degree vertices of the spanning tree is the entire vertex set! Thus, finding a Steiner cycle for these vertices is no easier than finding a TSP tour. However, in this example, we can see that there are many

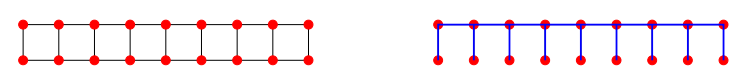

Fig. 4. A graph $G$ and a spanning tree.

other possible spanning trees. Figure 5 shows two other possible spanning trees and corresponding Steiner cycles. We note that given a spanning tree, the Steiner

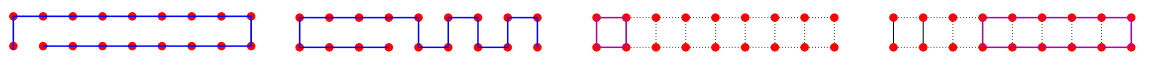

Fig. 5. Alternative spanning trees for $G$ and corresponding Steiner cycles.

cycle including the odd-degree nodes may not be unique. Another example of a graph $G$ and a spanning tree in which every vertex can have odd degree is shown in Figure 6. But, again, there are many other spanning trees in which only a subset of the vertices have odd degree.

Each of the examples we have considered so far actually contains a Hamiltonian path. Thus, by applying Theorem 2, we can see that they have a TSP tour of length at most $4 n / 3$. There are actually interesting examples of cubic, 3-edge connected graphs that do not contain a Hamiltonian path. The graph shown in Figure 8 is such a graph due to Zamfirescu [27]. We see that we can construct a spanning tree and Steiner cycle containing all of the vertices that have odd degree in the spanning tree. In conclusion, let us consider the following question: Suppose the standard linear programming relaxation for Graph TSP has value $n$ on a fixed graph. Then is there a spanning tree $T$ and a simple cycle $C_{T}$ that contains all of the vertices that are odd-degree in $T$ ? If a graph is Hamiltonian, then this is (trivially) true for any spanning tree. 

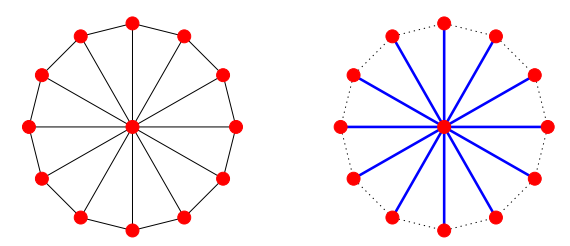

Fig. 6. The wheel graph has a spanning tree in which all vertices have odd degree.

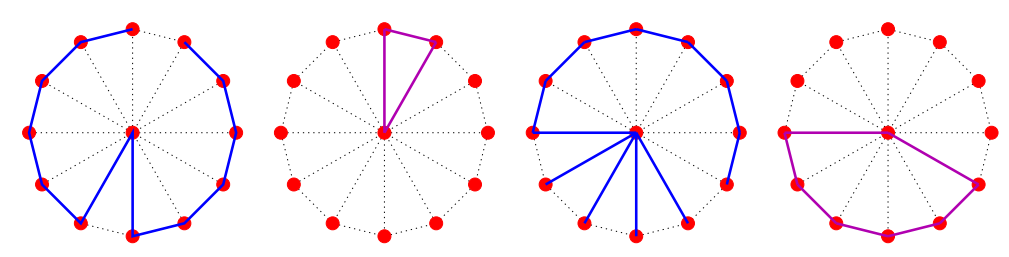

Fig. 7. Alternative spanning trees with fewer odd-degree vertices for the wheel graph.
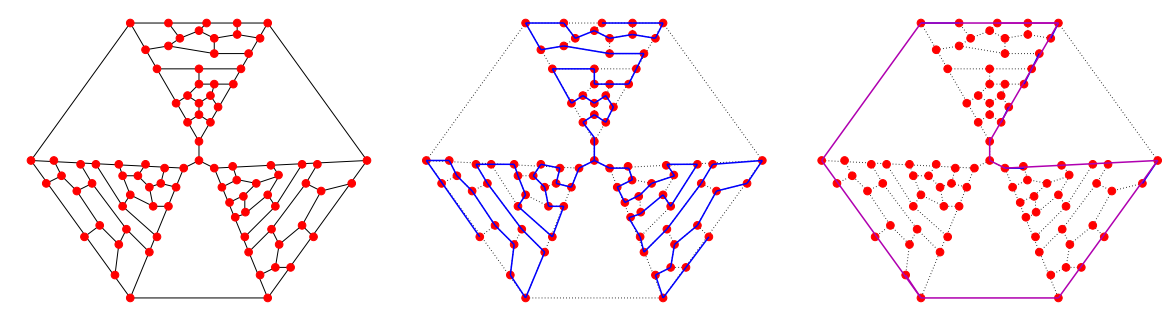

Fig. 8. A cubic, 3-edge connected graph with no Hamiltonian paths. We show a spanning tree and a corresponding Steiner cycle containing all the nodes with odd degree in the spanning tree.

\section{References}

1. N. Aggarwal, N. Garg, and S. Gupta. A 4/3-approximation for TSP on cubic 3-edge-connected graphs. arXiv preprint arXiv:1101.5586, 2011.

2. R. E. Aldred, S. Bau, D. A. Holton, and B. D. McKay. Cycles through 23 vertices in 3-connected cubic planar graphs. Graphs and Combinatorics, 15(4):373-376, 1999.

3. J. A. Bondy and U. S. R. Murty. Graph theory with applications, volume 290. Macmillan London, 1976.

4. S. Boyd, R. Sitters, S. van der Ster, and L. Stougie. TSP on cubic and subcubic graphs. In Integer Programming and Combinatoral Optimization, pages 65-77. Springer, 2011.

5. N. Christofides. Worst-case analysis of a new heuristic for the travelling salesman problem. Technical report, DTIC Document, 1976.

6. V. Chvatal. New directions in Hamiltonian graph theory. New directions in graph theory ed. by F. Harary, Academic press, NY London, 1973. 
7. V. Chvátal and P. Erdös. A note on Hamiltonian circuits. Discrete Mathematics, 2(2):111-113, 1972.

8. G. Cornuéjols, J. Fonlupt, and D. Naddef. The traveling salesman problem on a graph and some related integer polyhedra. Mathematical programming, 33(1):1-27, 1985.

9. G. Dirac. Some theorems on abstract graphs. Proceedings of the London Mathematical Society, 3(1):69-81, 1952.

10. G. Dirac. Short proof of Menger's graph theorem. Mathematika, 13(1):42-44, 1966.

11. I. Fournier. Cycles et numérotations de graphes. These d'Etat, LRI, Université de Paris-Sud, 1985.

12. S. O. Gharan, A. Saberi, and M. Singh. A randomized rounding approach to the traveling salesman problem. In Foundations of Computer Science (FOCS), 2011 IEEE 52nd Annual Symposium on, pages 550-559. IEEE, 2011.

13. J. J. S. González. The Steiner cycle polytope. European Journal of Operational Research, 147(3):671-679, 2003.

14. S. Gupta. Towards a $\frac{4}{3}$-approximation for the metric traveling salesman problem. Master's thesis, Indian Institute of Technology, Delhi, May 2011.

15. N. Guttmann-Beck, R. Hassin, S. Khuller, and B. Raghavachari. Approximation algorithms with bounded performance guarantees for the clustered traveling salesman problem. Algorithmica, 28(4):422-437, 2000.

16. D. A. Holton, B. D. McKay, M. D. Plummer, and C. Thomassen. A nine point theorem for 3-connected graphs. Combinatorica, 2(1):53-62, 1982.

17. J. Hoogeveen. Analysis of Christofides' heuristic: Some paths are more difficult than cycles. Operations Research Letters, 10(5):291-295, 1991.

18. K. Menger. Zur allgemeinen Kurventheorie. Fundamenta Mathematicae, 10(1):96$115,1927$.

19. T. Mömke and O. Svensson. Approximating graphic TSP by matchings. In IEEE 52nd Annual Symposium on Foundations of Computer Science, pages 560-569, 2011.

20. M. Mucha. $\frac{13}{9}$-approximation for graphic TSP. Theory of Computing Systems, pages 1-18, 2012.

21. K. Ozeki and T. Yamashita. A degree sum condition concerning the connectivity and the independence number of a graph. Graphs and Combinatorics, 24(5):469483, 2008.

22. A. Sebő. Eight-fifth approximation for the path TSP. In Integer Programming and Combinatorial Optimization, pages 362-374. Springer, 2013.

23. A. Sebö and J. Vygen. Shorter tours by nicer ears: $7 / 5$-approximation for graphic TSP, $3 / 2$ for the path version, and $4 / 3$ for two-edge-connected subgraphs. arXiv preprint arXiv:1201.1870, 2012.

24. R. Shi. 2-neighborhoods and Hamiltonian conditions. Journal of graph theory, 16(3):267-271, 1992.

25. M. Steinová. Approximability of the minimum Steiner cycle problem. Computing and Informatics, 29(6+):1349-1357, 2012.

26. N. K. Vishnoi. A permanent approach to the traveling salesman problem. In Foundations of Computer Science (FOCS), 2012 IEEE 53rd Annual Symposium on, pages 76-80. IEEE, 2012.

27. T. Zamfirescu. Three small cubic graphs with interesting Hamiltonian properties. Journal of Graph Theory, 4(3):287-292, 1980. 